\title{
Italian population yields world's largest twin registry
}

To the editor - Twin registries are considered unique research resources ${ }^{1}$. Concordance rate studies in monozygotic vs. dizygotic twins and biological studies in identical twins (concordant or discordant for a disease) substantially contribute to the definition of genetic and non-genetic etiologic factors in multigenic/multifactorial illnesses. Twin registries in Denmark, Finland, Norway and Sweden have been particularly valuable for being population-based, thus covering all twins without enrollment biases. Nonetheless, owing to the relatively small population size of these countries, concordance rate studies or biological investigations in identical twins are likely not to include enough cases, especially in diseases with low prevalence. Study designs that may overcome these as well as other limitations require greater numbers of sub- jects ${ }^{2}$. Registries similar to those of the Scandinavian countries do not exist in more populous nations. In Italy there is a notable opportunity for the creation of a population-based twin registry: all living individuals receive an alphanumeric identification code, used so far for fiscal purposes only (so called "fiscal code"). The code identifies the individual's last and first names, and date and place of birth. Subjects with identical family name, date of birth and place of birth are potential twins. Importantly, through tax return forms it is also possible to trace the individual's present residence. Previous requests to the Ministero delle Finanze (the Italian finance ministry) to use this database to create a twin registry met with difficulties related to restrictive laws on the access to personal information. The last European directive on confidential

\section{Clear about cancer therapies}

To the editor - We were surprised and disappointed by the tack taken by Lamb and Friend in their News \& Views article' concerning a study by Waldman et al. addressing the role of the p21 checkpoint in determining clonogenic cell survival and tumor curability following ionizing radiation (IR) therapy $y^{2}$. A major thrust of the new p21 work is an uncoupling of the in vitro from the in vivo IR response of $\mathrm{p} 21 \%$ colon cancer cells to IR. Although Waldman et al. stated that results similar to those observed with IR were obtained with in vitro assays of cell survival after treatment with adriamycin and etoposide, these data were not shown. Indeed, to our knowledge no data have been published indicating that the in vivo p21 status-dependent effect seen with IR applies to any anticancer drug.

As Lamb and Friend rightly indicated, devising strategies to predict chemotherapy outcomes is a laudable goal. Not at all clear from their News \& Views piece, however, is the fact that the same goal has long been sought in IR research: predicting responses of cancers to radiation therapy (RT), a modality used in approximately half of all cancer sufferers in the western world ${ }^{3}$, has been referred to as the "Holy Grail" of radiation research".

We join Lamb and Friend in hoping that such new finding $s^{2}$ may prove generalizable to anti-cancer modalities in addition to IR. However, as the data presented by Waldman et al. are restricted entirely to IR, the emphasis of the News \& Views article should have been on the potential of this exciting new finding with regards to RT, rather than chemotherapy, for cancer.

1. Lamb, I \& Friend, S. Which guesstimate is the best guesstimate? Predicting chemotherapeutic outcomes Nature Med. 3, 962-963 (1997).

2. Waldman, T. et al. Cell-cycle arrest versus cell death in cancer therapy. Nature Med. 3, 1034-1036 (1997)

3. McKay, M \& Langlands, A. The American "Patterns of Care" study: A model for the assessment of the quality of patient care in Radiation Oncology. Aust. Radiol. 34, 306-311 (1990).

4. Peters. L. The ESTRO Regaud Lecture: Inherent radiosensitivity of tumor and normal tissue cells as a predic tor of human normal tissue response. Radiother. Oncol. 17, 177-190 (1990).

Michael J. McKay ${ }^{1,2} \&$ Lester J. Peters ${ }^{1}$

Division of Radiation Oncology $y^{i}$ and Research ${ }^{2}$

Peter MacCallum Cancer Institute

St. Andrews Place, Melbourne 3000, Australia data $\mathrm{a}^{3}$ - and the Italian legislation that followed it - now allows such access for scientific research. With appropriate safeguards, previous restrictions on the use of personal data for scientific purposes have been removed. The Italian Ministero delle Finanze has therefore authorized access to the fiscal code database for research purposes. Using a computerized record linkage algorithm based on the same last name, same place and same date of birth, it has been possible to identify the entire population of potential twins. Using this strategy, we identified about 1,600,000 individuals, a figure close to that expected from the Italian population size of about $57,000,000$ and a twin birth rate of $1: 80(=1,425,000)$. An excess of $175,000(=12 \%)$ was also expected due to identical codes of unrelated individuals with the most common family names. About $450,000(=30 \%)$ of the subjects are expected to represent monozygotic twins. This constitutes, to our knowledge, the world's largest twin population. The records are now available for matching with a number of centralized medical databases (hospital discharges, social sickness insurance for drug prescriptions, cancer registries and others) in order to select diseased individuals. The database is open to all researchers interested in using it. An oversight committee will ensure that the twins' privacy is protected against unwarranted intrusion. The identification of the Italian twin population will provide an opportunity for investigators in many fields of medical science.

1. Hrubec, Z, Robinette C.D. The study of human twins in medical research. N. Engl. 1. Med. 310, 435-441 (1984)

2. Phillips, D.I.W. Twin studies in medical research: can they tell us whether diseases are genetically determined? Lancet 341, 1008-1009 (1993)

3. Lynge, E. New draft on European directive on confidential data. At last a step forward for epidemiological research. B.M.J. 310, 1024 (1995)

M. SAlvetTi ${ }^{1}$, G. Ristori' ${ }^{1}$, R. TOSI ${ }^{2}$,

C. FIESCHI ${ }^{1} \&$ A. STAZI

'Dipartimento di Scienze

Neurologiche-Università "La Sapienza"

o0185-Rome, Italy

${ }^{2}$ Consiglio Nazionale delle Ricerche

Istituto di Biologia Cellulare, 00137-Rome, Italy

${ }^{3}$ Istituto Superiore di Sanità, 00185-Rome Italy 